\title{
ELECTRON ACCELERATION IN SOLAR FLARES
}

\section{WOLFGANG DRÖGE* and PETER MEYER}

Enrico Fermi Institute, University of Chicago, 933 East 56th Street, Chicago, IL 60637, U.S.A.

\section{PAUL EVENSON}

Bartol Research Institute, University of Delaware, Newark, DE 19716, U.S.A.

and

\section{DAN MOSES}

American Science and Engineering Inc., Fort Washington, Cambridge, MA 02139, U.S.A.

\begin{abstract}
For the period September 1978 to December 1982 we have identified 55 solar flare particle events for which our instruments on board the ISEE-3 (ICE) spacecraft detected electrons above $10 \mathrm{MeV}$. Combining our data with those from the ULEWAT spectrometer (MPI Garching and University of Maryland) electron spectra in the range from 0.1 to $100 \mathrm{MeV}$ were obtained. The observed spectral shapes can be divided into two classes. The spectra of the one class can be fit by a single power law in rigidity over the entire observed range. The spectra of the other class deviate from a power law, instead exhibiting a steepening at low rigidities and a flattening at high rigidities. Events with power-law spectra are associated with impulsive ( $<1 \mathrm{hr}$ duration) soft X-ray emission, whereas events with hardening spectra are associated with long-duration ( $>1 \mathrm{hr}$ ) soft X-ray emission. The characteristics of long-duration events are consistent with diffusive shock acceleration taking place high in the corona. Electron spectra of short-duration flares are well reproduced by the distribution functions derived from a model assuming simultaneous second-order Fermi acceleration and Coulomb losses operating in closed flare loops.
\end{abstract}

\section{Introduction}

Energetic particle spectra observed in interplanetary space following solar flares are one measure of particle spectra at their solar acceleration sites. The study of these particles offers a unique opportunity to understand high-energy acceleration processes and to obtain information on the physical conditions at the flare site. In large solar flares, electrons are accelerated to energies of more than $100 \mathrm{MeV}$ and ions to energies of up to several $\mathrm{GeV}$.

In this paper we present an analysis of a recent study (Moses et al., 1989) of the shapes of solar flare electron spectra in the energy range $0.1-100 \mathrm{MeV}$. Previous surveys of electron flare spectra have been conducted by Datlowe (1971), Lin, Mewaldt, and van Hollebeke (1982), Evenson et al. (1984), and Cane, McGuire, and von Rosenvinge (1986). Compared to the earlier studies, our recent work has the advantage of a nearly continuous coverage over a four year period of high solar activity and a superior energy range and resolution.

It is found that solar flare electrons exhibit a variety of spectral shapes in the range from mildly relativistic to ultrarelativistic energies. When expressed as a function of rigidity, the spectra can be divided into two broad groups. The spectra of one group are

\footnotetext{
* Present address: Institut für Kernphysik, Kiel University, Olshausenstr. 40, D-2300 Kiel, F.R.G.
} 
consistent with power laws in rigidity, whereas the spectra of the other group sometimes become excessively hard above $5 \mathrm{MeV}$, an effect not noticed in previous observations. We find that our identification of two classes of spectral shapes correlates well with the classification scheme of interplanetary particle events based upon the 1-8 soft X-ray duration of the associated flare, as was suggested by Kahler et al. (1984) and Cane, McGuire, and von Rosenvinge (1986).

We have modeled the observed electron spectra by diffusive shock acceleration (Axford, Leer, and Skadron, 1977) and stochastic acceleration models including Coulomb losses (Steinacker, Dröge, and Schlickeiser, 1988). We find that for any given event, the stochastic acceleration model gives good fits. Diffusive shock acceleration is consistent with most of the long-duration soft $\mathrm{X}$-ray events, but fails to explain shortduration spectra.

\section{Data Analysis}

The observations were made with two instruments onboard the International Sun-Earth Explorer 3 (ISEE-3/ICE) spacecraft: the ULEWAT spectrometer (MPI/University of Maryland) which measured the electron flux in the energy range $0.075-1.3 \mathrm{MeV}$, and the University of Chicago MEH spectrometer which measured the electron flux in the energy range 5-100 MeV. The time interval of the survey extends from launch of ISEE-3 in August 1978 through December 1982. The ISEE-3 spacecraft was positioned at the Earth-Sun Lagrangian point well outside of the Earth's geomagnetic field.

The electron events for the survey were chosen in the following manner. All events which had a significant $(>3 \sigma)$ flux enhancement at energies above $10 \mathrm{MeV}$ and which could be associated with a flare were considered. To minimize propagation effects only events with a well-defined flux rise time were selected; events which were disturbed by an interplanetary shock were removed from the survey. A total of 55 events were found to satisfy these criteria.

For each event the electron spectrum was constructed by taking the maximum flux in each energy interval. It was shown by Lin, Mewaldt, and van Hollebeke (1982) that time of maximum spectra have the same shape and slope as the original injection spectra, if the propagation through the interplanetary medium can be described by standard diffusion treatment and if the observations are at energies sufficient to neglect the effects of convection and adiabatic deceleration.

A full description of the instruments, the analysis method used as well as a detailed table with the properties of each event will be presented elsewhere (Moses et al., 1989).

\section{Results}

Figure 1 shows the electron spectrum for the 19 December, 1982 event. In order to allow a direct comparison of the observed spectra with spectra predicted by shock and stochastic acceleration models we have plotted the electron flux density in energy space $J(E)$ which is proportional to the electron number density in momentum space $N(p)$, 


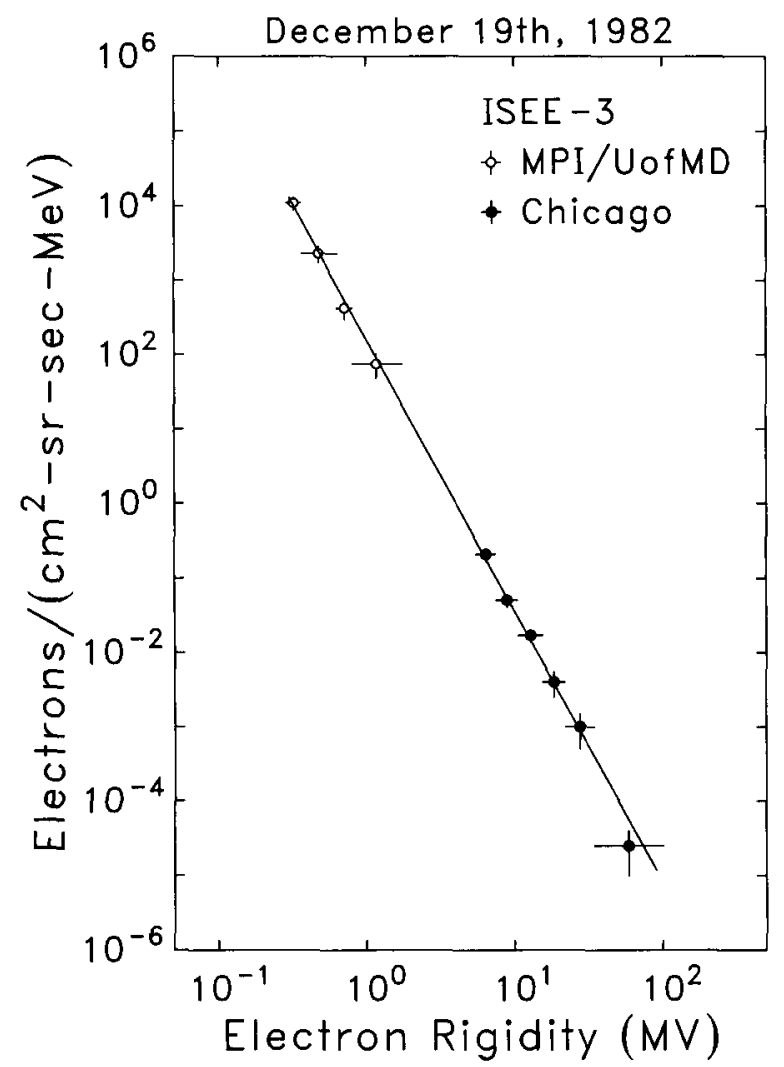

Fig. 1. Electron spectrum of the long-duration flare from 19 December, 1982. The spectrum is fitted by solution (4) with $p_{0}=0.3 \mathrm{MeV} \mathrm{c}^{-1}, p_{c}=0.1 \mathrm{MeV} \mathrm{c}^{-1}$, and $\alpha T=0.2$. A shock acceleration model with a compression ratio $r=2.1$ can reproduce the spectrum as well.

as a function of rigidity. As can be seen from Figure 1, the spectrum of this event can be well fitted by a single power law in rigidity (respectively momentum). Figure 2 shows the event of 7 June, 1980 ( $3: 12$ UT). The spectral shape of this event is quite different. A power-law spectrum is observed at rigidities above $\sim 5 \mathrm{MeV}$, but the spectrum becomes much steeper towards lower rigidities.

We find that, with regard to their spectral shapes, the electron events of our survey can be divided into two broad groups. The spectra of one group are reasonably well fitted by single power laws in rigidity, whereas the spectra of the other group deviate from single power laws: they are flatter at high rigidities and steeper at low rigidities. In order to find a classification scheme we have made separate power-law fits to the low- and high-rigidity part of each spectrum and we have determined the duration at $10 \%$ of the peak flux of the 1-8 $\mathrm{A}$ soft $\mathrm{X}$-ray emission of the parent flare. The ratio of the low- to the high-rigidity spectral index as a function of the soft $\mathrm{X}$-ray duration is shown in Figure 3. Events associated with long-duration soft X-ray emission (LDE) have spectral index ratios close to unity. This indicates that their spectra can be modeled 


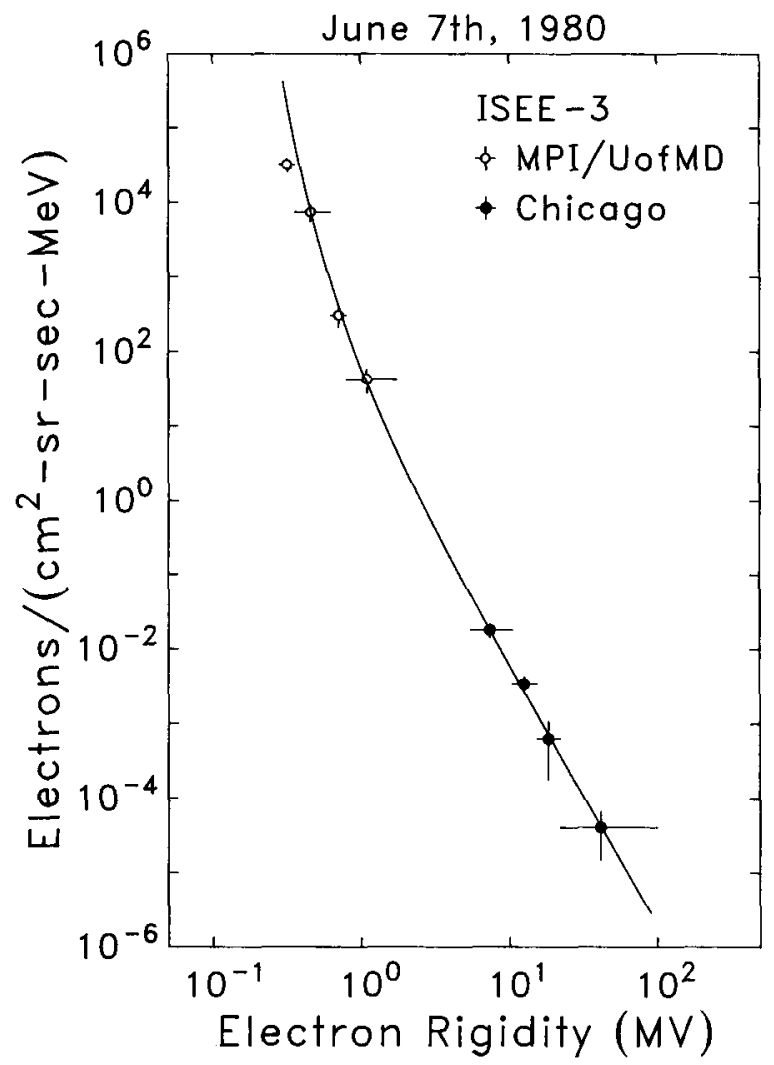

Fig. 2. Electron spectrum of the short-duration flare from 7 June, 1982 (3:12 UT). The spectrum is fitted by solution (4) with $p_{0}=0.3 \mathrm{MeV} \mathrm{c}^{-1}, p_{c}=0.93 \mathrm{MeV} \mathrm{c}^{-1}$, and $\alpha T=0.3$.

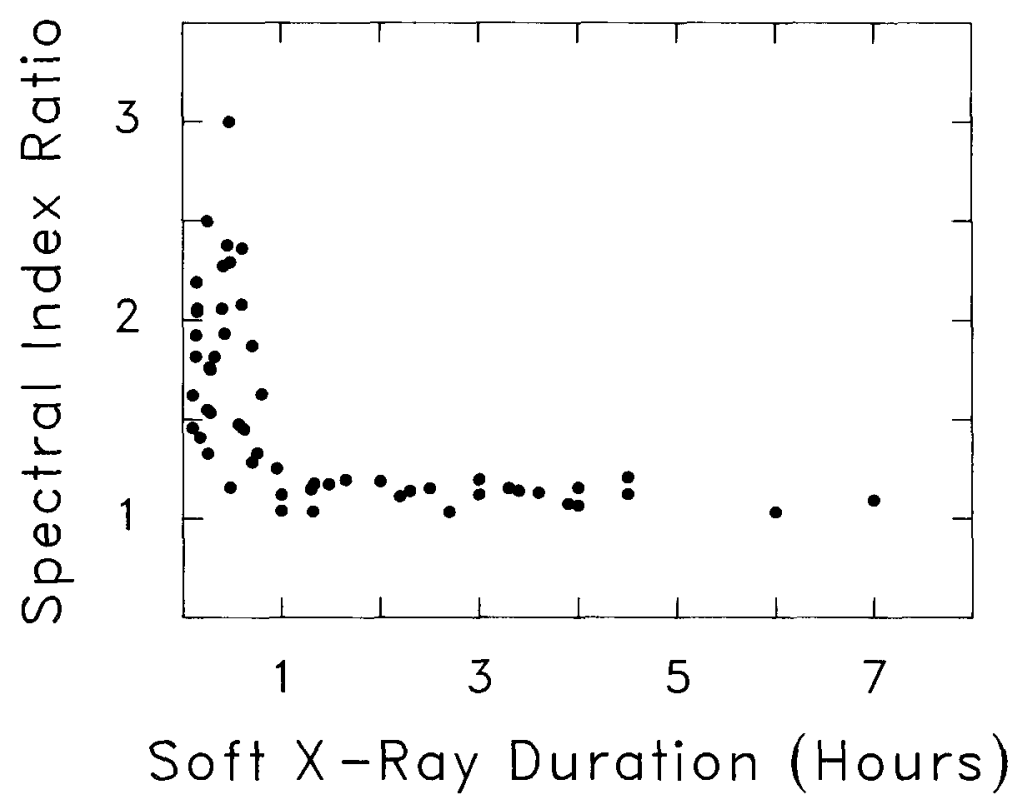

Fig. 3. Low-rigidity to high-rigidity spectral slope ratios plotted as a function of the soft X-ray duration. Events with a ratio $\approx 1$ can be fitted with a single power law. 
by one single power law over the entire range in rigidity; although some LDEs might exhibit a slight steepening at low rigidities. Events associated with short-duration soft $\mathrm{X}$-ray emission (SDE) have spectral index ratios well above unity, spectra of SDEs can, therefore, not be modeled by single power laws in rigidity. As can be seen from Figure 3, the events group into two distinct populations rather than exhibiting a continuous correlation between the spectral index ratio and the soft X-ray duration. The separation of the two populations occurs at roughly 1 hour. These results suggest that non-thermal (particle acceleration) and thermal (soft X-ray emission) features of a flare are related.

The classification of solar flare electron spectra is congruent with the classification of other flare characteristics with respect to their soft X-ray emission reported in earlier studies. The fact that soft X-ray events could be divided into two classes was first recognized by Pallavicini, Serio, and Vaiana (1977). They found that flares with short duration ( $\leq 1$ hour, Class I events) have smaller volumes, occur at lower heights $\left(\leq 10^{4} \mathrm{~km}\right.$ ) and have higher energy densities. In contrast, long duration (Class II) flares have larger volumes and lower energy densities and occur at greater heights $\left(\approx 5 \times 10^{4} \mathrm{~km}\right)$. Long-duration flares also are often associated with coronal mass ejections (Kahler et al., 1984) and interplanetary shocks (Cane, 1985), which in general is not the case for short-duration flares.

Evenson et al. (1984), studying electron events observed during 1980 which form a subset of the present survey, identified a class of events which correlate with gamma-ray flares and which have an unusually high abundance of electrons relative to protons. Figure 4 shows the electron to proton ratio as a function of the soft X-ray duration for the full data set. The electron flux has been evaluated at $14.5 \mathrm{MeV}$ from the power-law fits to the high-rigidity part of the spectrum and is compared with the proton flux in the 9-23 MeV range for all events where proton data are available (from Cane, McGuire, and von Rosenvinge, 1986). Although there is a large variability in the data, it can be seen from Figure 4 that the typical electron to proton ratio of SDEs events is approximately two orders of magnitude higher than that of LDEs. The higher electron to proton ratio for SDEs is due to the fact that they have much weaker proton fluxes, both classes have comparable electron fluxes. Again, the separation between the two classes occurs at a duration of roughly 1 hour.

\section{Discussion}

The fact that the spectra of long-duration events can be modeled by a single power law in rigidity indicates that one acceleration mechanism is operating over the whole observed energy range from 0.1 to $100 \mathrm{MeV}$. Stochastic acceleration in turbulent plasmas (cf. Ramaty, 1979) reproduces the observed spectra and is a mechanism commonly considered as candidate for the acceleration of relativistic solar flare electrons. On the other hand, a model involving acceleration at a shock wave passing through the corona can explain the spectra as well. Because LDEs are often associated with interplanetary shocks, a shock might be involved in particle acceleration in this class of flares. The observed spectral indices for LDEs are in the range 3.1-4.7, 


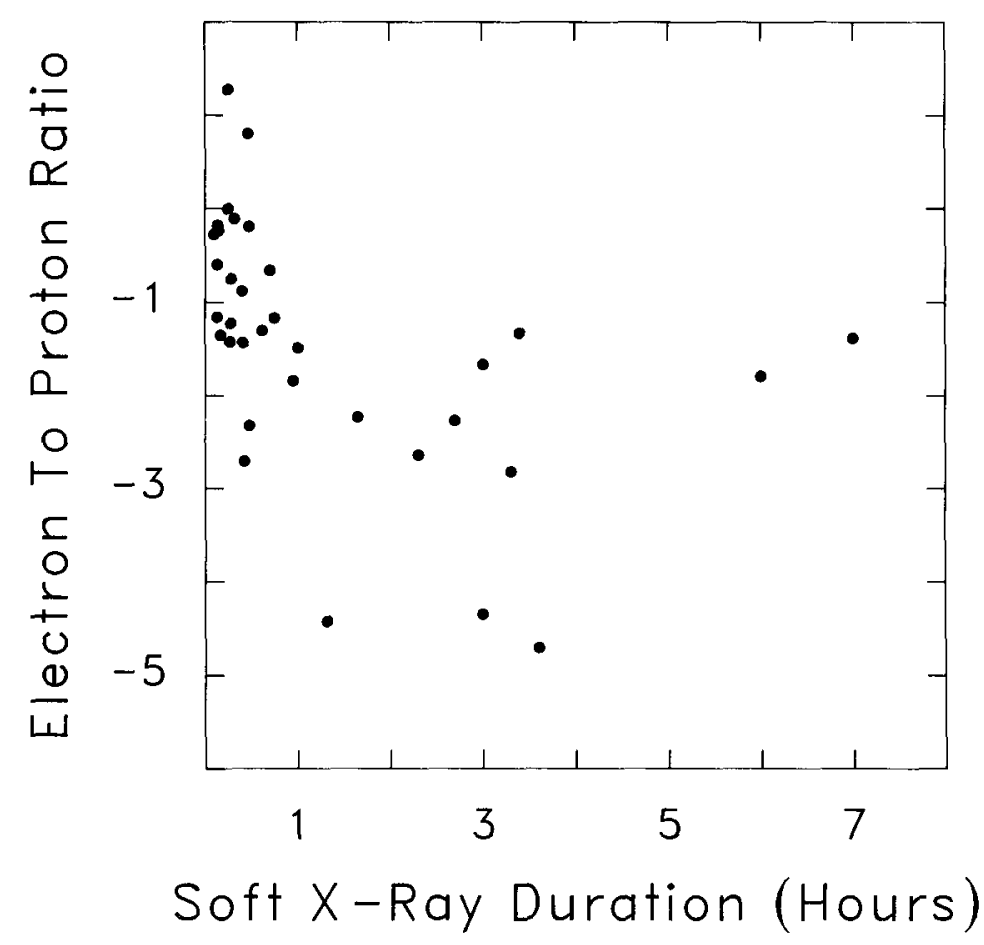

Fig. 4. Electron to proton flux ratios as a function of the soft X-ray duration.

corresponding to compression ratios in the range 1.8-2.4 in a diffusive shock acceleration model. The electron spectra alone cannot distinguish between these options.

There are several possible explanations for the spectral shape of short-duration events. A superposition of two components - a steep spectrum at low energies related to acceleration in the impulsive phase of the event, and a harder spectrum of particles accelerated in a second step/second phase - is one possibility. We note that simple diffusive shock acceleration cannot model the spectra of SDEs. However, if the backreaction process on shocks due to their acceleration of particles (Drury, 1984) is considered, the resulting spectrum can be of the form of SDE spectra. Back-reaction effects might be important for shocks in an environment with high energy densities, as can be expected for impulsive flares, but a detailed model involving an application of this effect on acceleration in solar flares is not yet available.

The model we have applied to fit the spectra of short-duration events is the one by Steinacker, Dröge, and Schlickeiser (1988), who demonstrated that stochastic acceleration taking into account ionization energy losses can produce the required steepening of the spectra at low rigidities. In this model it is assumed that electron acceleration in SDEs takes place in compact magnetic loops low in the corona where the plasma density is high and ionization losses cannot be neglected.

As was shown by Dröge and Schlickeiser (1986), the effects of stochastic accelera- 
tion, additional energy gain and loss processes and particle escape can be incorporated in a transport equation in momentum space

$$
\frac{\partial f}{\partial t}-\frac{1}{p^{2}} \frac{\partial}{\partial p}\left(p^{2} D(p) \frac{\partial f}{\partial p}\right)+\frac{1}{p^{2}} \frac{\partial}{\partial p}\left(p^{2}\left(\dot{p}_{G}+\dot{p}_{L}\right) f\right)+\frac{f}{T(p)}=Q(p),
$$

where $p$ is the particle momentum, $N(p)=4 \pi p^{2} f(p, t)$ the number of particles per unit momentum and volume, and $\dot{p}_{G}$ and $\dot{p}_{L}$ are the systematic gain and loss rates of the particles. The effects of particle escape out of the acceleration region have been combined in the escape time $T$, and $Q(p, t)$ represents sources and sinks of the particles. The momentum diffusion coefficient $D(p)$ depends on the nature of the stochastic process and is of the form

$$
D(p)=\frac{p^{2} \alpha}{3 \beta l},
$$

where $\alpha=V^{2} /(l c), \beta=v / c, V$ is the velocity of the fluid elements or the Alfvén velocity, $v$ is the particle velocity, $l$ is an effective mean free path against particle scattering off the plasma waves. The loss rate due to Coulomb collisions of electrons in a fully-ionized hydrogen plasma has been considered by Bai (1982). It was found that for reasonable flare parameters $\left(T \approx 10^{7} \mathrm{~K}, n_{e}=n_{p}=10^{10} \mathrm{~cm}^{-3}\right)$ the loss rate can be written as

$$
-\dot{p}_{\text {Coul }}=\frac{m c}{\tau_{L} \beta^{2}}
$$

$\left(\tau_{L}=0.146 \times 10^{13}\left(n \mathrm{~cm}^{-3}\right)^{-1} \mathrm{~s}\right)$ at all momenta of interest.

In order to obtain asymptotic power laws at high rigidities we have to make the assumption $\alpha T=$ const. In this case, the steady-state solution of Equation (1) for monoenergetic injection $Q(p) \sim \delta\left(p-p_{0}\right)$ is of the form

$$
N\left(p>p_{0}\right) \sim p^{1 / 2-\mu} M\left(\mu-\frac{1}{2}, 1+2 \mu, \frac{4 p_{c}}{p}\right),
$$

where $M$ is the Kummer function or confluent hypergeometric function (Abramowitz and Stegun, 1965), $\mu=\sqrt{\frac{9}{4}+3 / \alpha \mathrm{T}}$, and $p_{c}=3 m c /\left(4 \alpha \tau_{L}\right)$ is a characteristic momentum where the gain rate due to stochastic acceleration and the loss rate are equal.

We have made fits to all electron spectra with both a single power law in rigidity and the Kummer function solution (4) and applied a $\chi^{2}$-test for the goodness of the fit. For the long-duration events, both spectral forms provide good fits to the data (cf. Figure 1). For short-duration events, single power laws generally give poor fits. Here the Kummer function solution improves the quality of the fits drastically, giving fits with values for $\chi^{2}$ per degree of freedom similar to the ones obtained for long-duration events (cf. Figure 2).

Events which are modeled by a single power law require one fit parameter, i.e., the compression ratio in a diffusive shock acceleration model or the acceleration parameter 


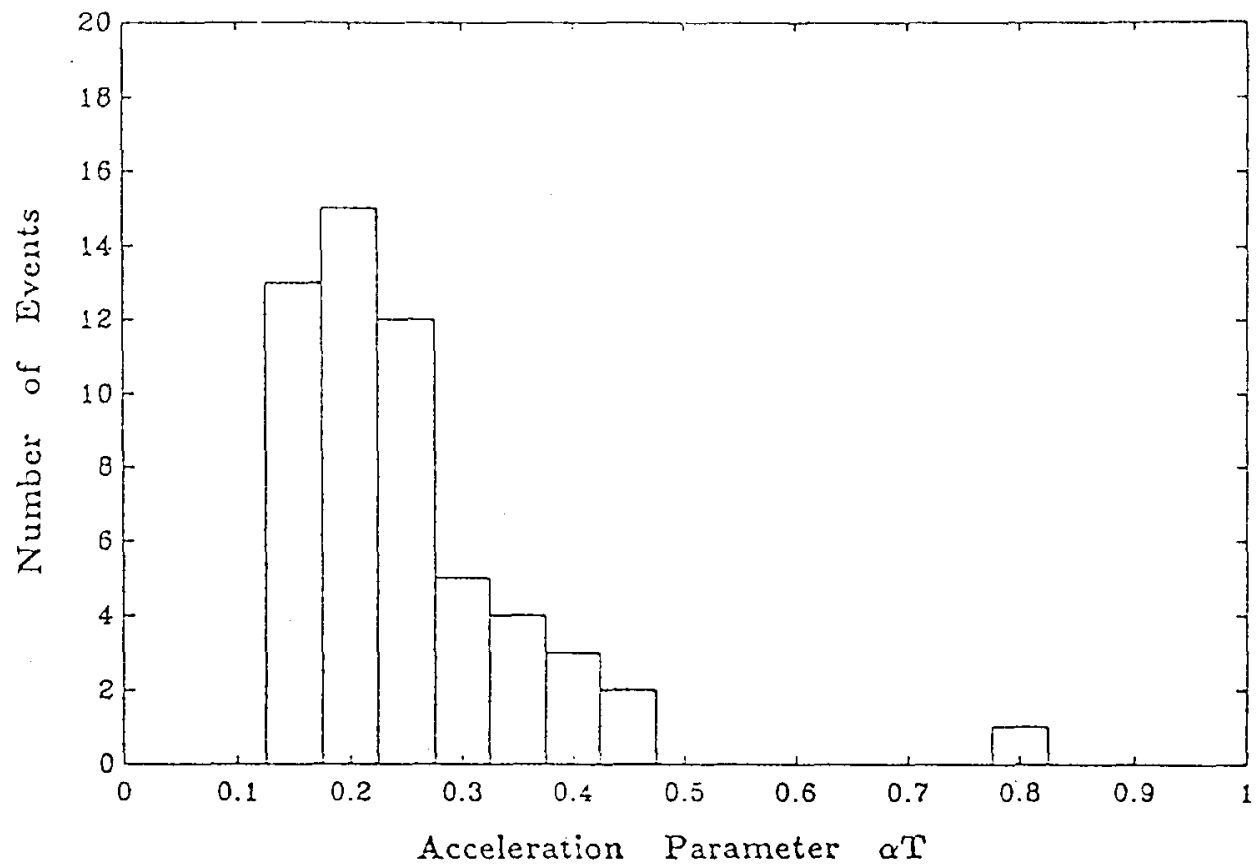

Fig. 5. Distribution function of the stochastic acceleration parameter $\alpha T$. The event with $\alpha T=0.8$ is the flare of 29 June, 1980 which had an exceptionally hard spectrum at high rigidities.

$\alpha T$ for stochastic acceleration without losses. In a stochastic acceleration model which includes ionization losses, the additional fit parameter is the characteristic momentum $p_{c}$. This parameter describes where the spectrum begins to deviate from the asymptotic power law at high rigidity and turns into a steepening towards lower rigidity.

In Figure 5 we show the distribution of the fit parameter $\alpha T$ for all events of our survey. Values for $\alpha T$ are in the range 0.12 to 0.8 . As mentioned above, at high rigidities the spectra of short-duration events are harder than the spectra of long-duration events. The average value of $\alpha T$ is 0.19 for SDEs, whereas it is 0.3 for LDEs. The values of the second fit parameter range from $p_{c} \approx 3 \mathrm{MeV} \mathrm{c}^{-1}$ required to model events where the spectral steepening is most pronounced, to $p_{c} \rightarrow 0$ in which case the Kummer function solution becomes a power law over the entire observed range to fit LDE spectra. We are in the process of studying possible correlations between the two fit parameters and between the fit parameters and observables of the parent flares (Dröge et al., 1989). We note that the values of $\alpha T$ we obtain for the spectra of our survey are on average one order of magnitude larger than values of $\alpha T$ required to fit proton spectra with stochastic acceleration (cf. McGuire and von Rosenvinge, 1984). This might indicate that protons interact with different plasma waves or have different escape times.

We believe that stochastic acceleration including ionization losses is a promising model for the acceleration of energetic electrons in impulsive solar flares, although other explanations are possible. This model also provides good fits for long-duration events, but a further study of the properties of each class of flares is required to decide whether 
the same acceleration mechanism operating under different boundary conditions or rather two distinct acceleration mechanisms are more likely to accelerate electrons in impulsive and long-duration flares.

\section{Acknowledgements}

We are grateful to Dr Dietrich Hovestadt for making available the electron data from the ULEWAT experiment. We wish to thank Leo Krawczyk for invaluable assistance in the data analysis. This work was supported in part by NASA contracts NAS5-28500 and NAS5-25496. W.D. gratefully acknowledges support as a Lynen Fellow of the Alexander von Humboldt-Stiftung.

\section{References}

Abramowitz, M. and Stegun, I. A.: 1965, Handbook of Mathematical Functions, Dover, New York. Axford, W. I., Leer, E., and Skadron, G.: 1977, Proc. 15th Int. Cosmic Ray Conf. (Plovdiv) $11,132$. Bai, T.: 1982, Astrophys. J. 259, 342.

Cane, H. V.: 1985, J. Geophys. Res. 90, 191.

Cane, H. V., McGuire, R. E., and von Rosenvinge, T. T.: 1986, Astrophys. J. 301, 448.

Datlowe, D.: 1971, Solar Phys. 17, 436.

Dröge, W. and Schlickeiser, R.: 1986, Astrophys. J. 305, 909.

Dröge, W. et al: 1989 (in preparation).

Drury, L. O'C.: 1984, Adv. Space Res. 4, 185.

Evenson, P., Meyer, P., Yanagita, S., and Forrest, D.: 1984, Astrophys. J. 283, 439.

Kahler, S. W., Sheeley, N. R., Howard, R. A., Koomen, R. J., Michels, D. J., McGuire, R. E., von Rosenvinge,

T. T., and Reames, D. V.: 1984, J. Geophys. Res. 89, 9683.

Lin, R. P., Mewaldt, R. A., and van Hollebeke, M. A. I.: 1982, Astrophys. J. 253, 949.

McGuire, R. E. and von Rosenvinge, T. T.: 1984, Adv. Space Res. 4, 117.

Moses, D., Dröge, W., Meyer, P., and Evenson, P.: 1989, Astrophys. J. (submitted).

Pallavicini, R., Serio, S., and Vaiana, G.: 1977, Astrophys. J. 216, 108.

Ramaty, R.: 1979, in J. Arons, C. Max, and C. McKee (eds.), Particle Acceleration Mechanisms in Astrophysics, AIP, No. 56, AIP, New York, p. 135.

Steinacker, J, Dröge, W., and Schlickeiser, R.: 1988, Solar Phys. 115, 313. 